\title{
Influence of electrocautery-induced electromagnetic interference on quantitative electroencephalographic monitoring of hypnosis during general anesthesia: comparison between the ADMS $^{\circledR}$ and the BIS VISTA ${ }^{\mathrm{TM}}$
}

\author{
Dae-hee Kim ${ }^{1}$, Ji-young Yoo ${ }^{1}$, Jong-Yeop Kim ${ }^{1}$, Soo-hwan Ahn ${ }^{1}$, \\ Seongsu Kim ${ }^{2}$, and Sang-kee Min ${ }^{1}$ \\ Department of Anesthesiology and Pain Medicine, ${ }^{1}$ Ajou University School of Medicine, Suwon, ${ }^{2} \mathrm{CHA}$ Bundang \\ Medical Center, CHA University, Seongnam, Korea
}

\begin{abstract}
Background: Hypnosis monitors analyze small-amplitude electrical signals transmitted from the brain that could be exposed to the electromagnetic field that occurs around the body during electrocautery (ECT). We investigated the influence of ECT on hypnosis monitoring during anesthesia.

Methods: We simultaneously monitored BIS and uCON during 50 gynecologic oncology surgeries. During the episodes of ECT, we compared the absolute difference (a-Diff) between the baseline index and the most deviated index after ECT over either 30-60 s (ECT30-60) or more than $60 \mathrm{~s}($ ECT $>60$ ) between the monitors. We also investigated the bias and the limits of agreement between the monitors.

Results: Between the two monitors, the a-Diff of ECT30-60 was 1.4 \pm 1.1 for the BIS, which was significantly greater than 0.6 \pm 0.9 for the $\mathrm{uCON}(\mathrm{P}=0.003)$, and the a-Diff of ECT $>60$ was $16.5 \pm 8.2$ for the BIS, which was also significantly greater than 1.4 \pm 1.3 for $\mathrm{uCON}(\mathrm{P}<0.001)$. The intra-monitor index differences showed that the BIS during ECT $>60$ was significantly greater than that during ECT30-60 ( $\mathrm{P}<0.001)$, but the uCON showed no significant difference between ECT30-60 and ECT $>60$ (P $=0.056$ ). The estimated bias between the monitors was $6.3 \pm 9.8$ and $95 \%$ limits agreement was -12.3 to 25.0 .

Conclusions: Prolonged ECT intervention might lead to spurious estimations of quantitative EEG indexes. Therefore, hypnosis should be clinically assessed in combination with scrutinized judgment of relevant clinical symptoms and signs for hypnosis.
\end{abstract}

Keywords: Anesthesia; Electrocoagulation; Electroencephalography; Hypnosis.

Corresponding author: Sang-kee Min, M.D.

Department of Anesthesiology and Pain Medicine, Ajou University School of Medicine, 206 Worldcup-ro, Yeongtong-gu, Suwon 16499, Korea Tel: 82-31-219-5572, Fax: 82-31-219-5579, Email: anesmin@nate.com

ORCID: https://orcid.org/0000-0001-9796-1542

Received: June 2, 2017.

Revised: August 31, 2017 (1st); September 24, 2017 (2nd).

Accepted: September 25, 2017.

Korean J Anesthesiol 2018 October 71(5): 368-373

https://doi.org/10.4097/kja.d.18.27154

(c) This is an open-access article distributed under the terms of the Creative Commons Attribution Non-Commercial License (http://creativecommons.org/ licenses/by-nc/4.0/), which permits unrestricted non-commercial use, distribution, and reproduction in any medium, provided the original work is properly cited. 


\section{Introduction}

To prevent intraoperative awareness and assess patients' hypnotic states, various measures and indicators such as eyeball movement, pupillary reflex, arterial pressure, and sweating have traditionally been used during anesthesia [1,2]. However, it has been reported that these symptoms and signs do not assess the overall depth of anesthesia $[3,4]$.

Since the early 1990s, owing to advances in modern computer technology and complex statistical modeling techniques, raw electroencephalogram (EEG) signals have been easily analyzed and processed to be transformed as quantitative, dimensionless numbers that range from 0 to 99 or 100 that indicate the quantitative EEG indexes (qEEGi) used for hypnosis status during anesthesia. A bispectral index (BIS) monitor (BIS VISTA ${ }^{\mathrm{TM}}$, Aspect Medical Systems, Inc., USA) provides clinicians with a qEEGi of the BIS using bispectrum power analysis and nonlinear mixed-effects modeling [5], and maintaining a BIS between 40 and 60 has been reported to decrease the incidence of intraoperative awareness [6-8].

Recently, the $\mathrm{ADMS}^{\circledR}$ (Unimedics CO., Korea) monitor has been developed and introduced into clinical settings. This monitor provides a uCON of the qEEGi using mono-spectral power analysis and an Adaptive Neural Fuzzy Inference System (ANFIS), identical to the qCON index (Quantium Medical, Spain). Moreover, the $\mathrm{ADMS}^{\circledR}$ was made to be more stable while minimizing electrical interference and offering a more convenient graphical user interface, which the qCON has been reported to maintain acceptable correlation with the BIS [9].

However, as with any monitor, special issues affect these qEEG monitoring processes, and many factors have been reported as artifacts that could mislead the qEEGi [10]. During anesthesia, various electrical devices that produce direct electrical disturbance or electromagnetic interference have been reported to result in qEEGis that indicate incorrect hypnotic status. In particular, electrocautery (ECT), which is frequently used for surgical procedures such as cutting and coagulation, produces an electromagnetic field around the body and is reported to indicate paradoxically high BIS values [11]. It has also been reported that the BIS is not proportional to the effect-site concentration of propofol during ECT [12].

The purpose of this study was to determine the influence of ECT on the qEEGis from both monitors and their different behaviors by ECT duration for surgical procedures. We also investigated the bias and the agreement between the indexes during anesthesia.

\section{Materials and Methods}

After we obtained approval from the Institutional Review
Board and written informed consent from the patients, we enrolled 50 adult female patients with American Society of Anesthesiologists physical status 1-3, who were scheduled for gynecologic oncology surgeries in this study. The surgeries were extensive-multidisciplinary, combined with urologic and/or colorectal surgery, and expected to frequently use ECT. We excluded patients under age 16 or over age 80 , patients with neurologic, psychiatric, or endocrine disorders, and patients who were taking psychoactive drugs.

We simultaneously employed two monitors, BIS VISTA ${ }^{\mathrm{TM}}$ (Aspect Medical Systems, Inc., USA) for the bispectral index (BIS) and $\mathrm{ADMS}^{\circledR}$ (Unimedics CO., Korea) for the unicon index $(\mathrm{uCON})$. We set the current clocks of the monitors to synchronize to permit discrepancies of $1 \mathrm{~s}$ or less between two monitors. We set both the BIS smoothing rate and the uCON data averaging processing time to $15 \mathrm{~s}$, and we saved real-time data at every second for the post hoc analysis. Before we induced anesthesia, we attached each monitor's electric sensors to the patients' foreheads as described in their package inserts; we placed the two sensors opposite to each other (Fig. 1), randomly attaching them in one of four positions, contralateral and upper or lower.

We monitored all patients for standard anesthesia measures including electrocardiography, pulse oximetry, end-tidal gas, temperature, invasive blood pressure, and noninvasive cardiac output. After acquiring the pass to check the sensor's impedance, we induced anesthesia with $1.5-2.0 \mathrm{mg} / \mathrm{kg}$ boluses of propofol and targeted remifentanil effect-site concentrations of 3.0-4.0 $\mathrm{ng} / \mathrm{ml}$. For neuromuscular blockade, we administered $0.6 \mathrm{mg} / \mathrm{kg}$ of rocuronium and performed tracheal intubation. We ventilated the lungs with $50 \%$ air in oxygen and maintained anesthesia with 1.0-3.0\% sevoflurane and target-controlled remifentanil infusions, which were titrated to maintain BIS between 40 and 60 . When the BIS decreased to be below 35 or increased above 65 more than $5 \mathrm{~min}$, the sevoflurane concentration was changed accordingly.

During the surgeries, the surgeon used electrocautery $\left(\mathrm{FX}^{\mathrm{TM}}\right.$ Electrosurgical Generator, Valleylab Inc., USA) in unipolar

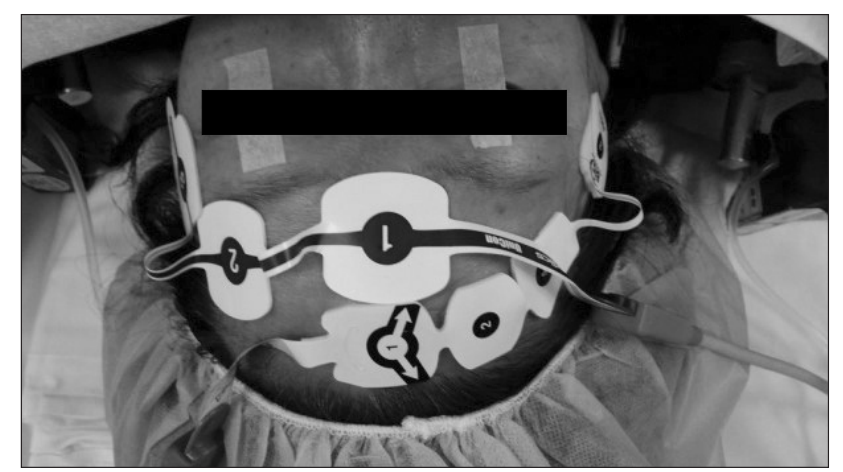

Fig. 1. Placement of the BIS and uCON sensors on a patient's forehead. 
(monopolar) mode for the surgical procedures of coagulation or cutting; we applied the grounding plate to the left buttock and set the ECT output power to $50 \mathrm{~W}$. The ECT starting and ending times were saved in the $\mathrm{ADMS}^{\circledR}$ monitor's memory using the event-mark function. When dose of sevoflurane and remifentanil changed, we excluded from the data analysis the episodes of ECT applied 10 min before and after these dosage changes. We also excluded ECT episodes that used output power at other than $50 \mathrm{~W}$ from the post hoc analysis.

The primary outcome measures of the study were the index differences during ECT between the two monitors and the intramonitor differences between the durations of ECT. After the end of anesthesia, we conducted postoperative data analysis. We defined the index at the moment ECT began as "pre-ECT" and defined the maximally deviated index from pre-ECT until $30 \mathrm{~s}$ after the ECT ceased as "post-ECT." We compared the absolute differences $(\mathrm{a}-\mathrm{Diff}=\mid$ pre-ECT - post-ECT $\mid$ ) between the monitors. Pilot analysis and our clinical experience showed occasional obscure and abnormal qEEGis when ECT continued for more than $1 \mathrm{~min}$. Therefore, we designed this study to investigate the different aspect changes in both indexes during 30-60 s of ECT (ECT30-60) and more than $1 \mathrm{~min}$ of ECT (ECT > 60).

To analyze estimated bias and paired-index agreement between the two qEEGis during surgery, we compared BIS and $\mathrm{uCON}$ using the Bland-Altman plot of (BIS $+\mathrm{uCON}) / 2$ for BIS - uCON with estimated bias and standard deviation (SD). The 95\% limits of agreement are expressed as estimated bias \pm $1.96 \times \mathrm{SD}$ [13]. We selected the paired-point indexes during the surgery, from skin incision to skin closure, and we also chose the data that had signal quality indexes (SQIs) more than 90 on both monitors and had the BIS artifact value of 0 as convincing signals for the analysis.

All data are expressed as mean \pm standard deviation (SD). We used SPSS 13.0 for Windows (SPSS Inc., USA) for the statistical analyses. We compared the ECT30-60 and ECT $>60$ between the monitors using the paired sample $t$ test and analyzed the intra-monitor differences between ECT30-60 and ECT > 60 using the independent-sample $t$ test, and we considered $\mathrm{P}<0.05$ to be statistically significant.

\section{Results}

The mean anesthesia duration was $5.2 \pm 2.1 \mathrm{~h}$, and the mean patient age was $54.7 \pm 12.6 \mathrm{yr}$ separately. The mean height was $152.2 \pm 5.3 \mathrm{~cm}$ and the weight was $59.3 \pm 7.6 \mathrm{~kg}$. There was no statistically significant difference in the position of each sensor: there were 11 cases for the Rt upper BIS/Lt lower ADMS, 12 cases for the Rt lower BIS/Lt lower ADMS, 14 cases for the Lt upper BIS/Rt lower ADMS, and 13 cases for the Lt lower BIS/Rt upper ADMS.
Table 1. Absolute Differences (a-Diff) in BIS and uCON between before and after Electrocautery (ECT)

\begin{tabular}{lcc}
\hline a-Diff & ECT30-60 $(\mathrm{n}=55)$ & ECT $>60(\mathrm{n}=38)$ \\
\hline BIS & $1.4 \pm 1.1^{*}$ & $16.5 \pm 8.2^{*,+}$ \\
uCON & $0.6 \pm 0.9$ & $1.4 \pm 1.3$ \\
\hline
\end{tabular}

Values are mean \pm SD. The ECT durations were 30 to $60 \mathrm{~s}$ for ECT3060 and over $60 \mathrm{~s}$ for ECT $>60 .{ }^{*} \mathrm{P}<0.05$ compared with uCON. ${ }^{\dagger} \mathrm{P}<0.05$ compared with ECT30-60.

Most duration of ECT lasted less than $30 \mathrm{~s}$. We observed 55 ECT30-60 episodes in 35 of the surgeries, and the mean duration of ECT30-60 was $47.0 \pm 8.1$ s. We also observed 38 episodes of ECT $>60$ in 24 surgeries, and the mean duration of ECT $>60$ was $135.0 \pm 49.7 \mathrm{~s}$.

During ECT30-60, the BIS a-Diff was $1.4 \pm 1.13$, and this was significantly greater than the $0.6 \pm 0.9$ for $\mathrm{uCON}(\mathrm{P}=0.003)$, but the mean a-Diff between BIS and ECT was only 0.79 (Table 1). During ECT $>60$, the a-Diff was $16.5 \pm 8.2$ for BIS, and this was significantly greater than the $1.4 \pm 1.3$ for $\mathrm{uCON}(\mathrm{P}<0.001)$. The ECT $>60$ BIS was also significantly greater than the ECT30-60 $(\mathrm{P}<0.001)$, but $\mathrm{uCON}$ showed no significant difference between ECT30-60 and ECT $>60(\mathrm{P}=0.056)$. We observed the maximal difference in the ECT $>60$ of BIS, where the BIS had been stable at 32 before the ECT, but it increased to 76 after the ECT.

In particular, the abrupt abnormal BIS index variations in the 38 episodes of ECT $>60$ showed three different patterns. First, the BIS abruptly increased after we used ECT ( 22 of 38 episodes, $58.9 \%)$. Second, the BIS abruptly decreased after we used ECT (13 of 38 episodes, $34.2 \%$ ). Third, the BIS abruptly increased and decreased or decreased and increased after we used ECT ( 3 of 38 episodes, 7.9\%). Fig. 2 shows three examples of actual snapshots of BIS and SQI time courses in each abnormal pattern.

Fig. 3 presents the Bland-Altman plots for $(\mathrm{BIS}+\mathrm{uCON}) / 2$ vs. (BIS - uCON), which contained 99,215 points of index pairs. The estimated bias was 6.3 , and the SD was 9.52 . The $95 \%$ limits of agreement (estimated bias $\pm 1.96 \times \mathrm{SD}$ ) were -12.3 to 25.0 .

\section{Discussion}

Short employments of ECT less than 1 min have shown minimal qEEGi differences in BIS and uCON between before and after ECT, and prolonged employments longer than $1 \mathrm{~min}$ also had minimal index uCON differences. However, under ECT longer than $1 \mathrm{~min}$, the BIS showed different patterns of abrupt change, and the difference in BIS was significantly greater than the $\mathrm{uCON}$.

The two monitors do have common features: they provide the suppression ratios and SQIs from the time domain, and both perform power analyses from the frequency domain following fast Fourier transforms. However, there are also differences 

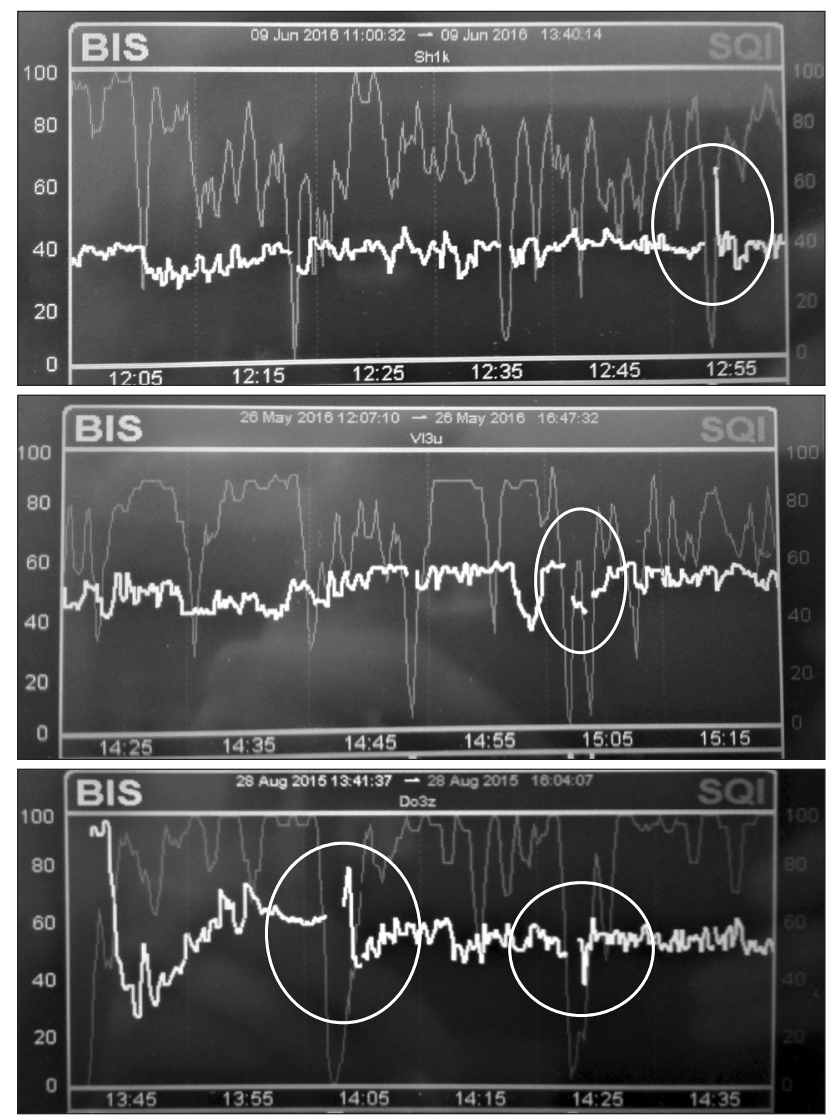

Fig. 2. Snapshots of the BIS (white spline graph) and SQI (gray spline graph) time courses show three typical abnormal BIS behaviors during ECT $>60$ that are highlighted in the white circles. The BIS index showed an abnormal rapid increase (upper snapshot), a rapid decrease (middle snapshot), and rapid increase and decrease or decrease and increase (lower snapshot) during ECT $>60$.

between the two monitors. BIS provides relative- $\beta$ ratios in the mono-spectral domain and SynchFastSlow in the bi-spectral domain, and these parameters are translated to qEEGi using non-linear mixed-effects modeling. However, uCON is based on mono-spectral power analysis, and four EEG spectral ratios at four different ranges frequencies are translated using ANFIS modeling. Both monitors also calculate EMG power expressed as $\mathrm{dB}$, but the reference power values and target frequency ranges for EMG differ. The EMG bar graph of the BIS displays the power (in decibels) in the frequency range of $70-110 \mathrm{~Hz}$, but the target frequency range EMG in the $\mathrm{uCON}$ is $30-45 \mathrm{~Hz}$. The precise algorithms for both modeling techniques are not open source or disclosed to the public, and for this reason we could not interpret the abnormal BIS behavior after the SQI fell to 0 following prolonged ECT and the subsequent SQI increased even though the artifacts from the ECT remained.

During anesthesia, many artifacts are known to influence qEEGi. Based on the original artifact sites, they are classified

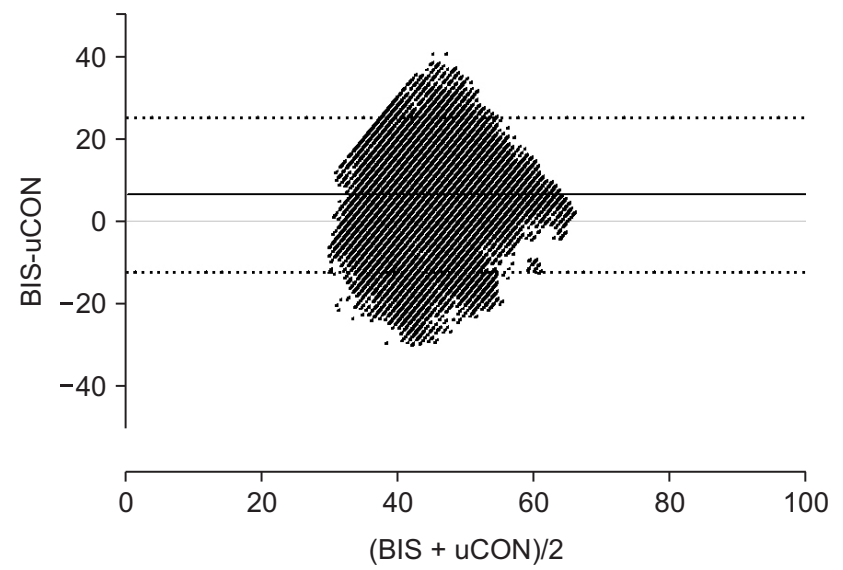

Fig. 3. The Bland-Altman plot for (BIS $+\mathrm{uCON}) / 2$ vs. (BIS - uCON) contains 99,215 paired-index points recorded for the 50 patients, reflecting the agreement between BIS and uCON. The thin solid line indicates the similarity of the two indexes, the thick solid line indicates the estimated bias of the two indexes, and the dotted lines show the $95 \%$ limits of agreement.

into three major categories: (1) artifacts from outside the head; (2) artifacts from within the head but outside the brain, EMG; and (3) artifacts from within the brain, atypical or pathologic EEGs [14]. Artifacts from outside the head have been reported to result from atrial pacers [15], warming blanket/convective air-warming systems [16-18], endoscopic shavers [19], electromagnetic systems [11], and laryngeal electromyography monitoring with an EMG endotracheal tube [20]. Most of these papers reported increased BIS during or after ECT and are clinical case reports of one or two patients' findings. The manufacturer's guidebook for the BIS VISTA ${ }^{\mathrm{TM}}$ monitor also shows abrupt BIS increases. However, we could not find any study that reported abnormal decreases and combined increases and decreases during ECT. During pilot study, we just encountered abnormal increases in qEEGi during ECT. However, during the main study of 50 patients, some abnormal decreases or combined increases and decreases of BIS were encountered without any manifestations of changes in patients' hypnotic status.

Low-voltage small-amplitude EEG signals (voltage range unit: $\mu \mathrm{V}$ ) are highly vulnerable and easily contaminated by the high-frequency electromagnetic noise during ECT (voltage range unit: $\mathrm{mV}$ ). Thus, as soon as ECT begins, the SQI begins to fall. The SQI indicates the percentage of time during which the pure EEG signals vs. other abnormal noise caused by various signals during a recent time interval, which is commonly set to be $1 \mathrm{~min}$ in most monitors. Therefore, the SQIs from both monitors fell to 0 after more than one minute of ECT. Inspection of the real-time saved data file showed that after SQI had fallen to zero, the ADMS algorithm was not likely to estimate any additional indexes, and the SQI was maintained at 0 until the ECT 
ceased. However, 3-4 s after the SQI had fallen to zero, the BIS showed abrupt increases or decreases, and the SQI alone began to increase, but the interference from ECT artifacts continued.

The details of versions of BIS monitor that we used in this study was hardware revision 4.00, platform 2.03, application 3.22. The BIS algorithm and hardware have been continuously developed and upgraded through a number of revisions [21]. The first version was released in 1992, and the most recently available version is BIS hardware platform v4.1; the upgraded versions of BIS that show a close correspondence with this study are v4.0 and 4.1. These versions were revised to make the BIS more resistant to ECT and EMG artifacts and to enhance the recognition and rejection of artifacts. However, when electromagnetic interference continues, the algorithm seems to continue processing the index while elevating the SQI. This forced BIS processing algorithm is difficult to understand and seems to be unreasonable to assess hypnosis during ECT.

In the Bland-Altman analysis of qEEGi data during anesthesia, although the BIS and the uCON were not identical, they did show good agreement. Jensen et al. [9] found that the bias between BIS and qCON was -2 , the SD was 12 , and the $95 \%$ of limits of agreement were calculated as -26 to 22 , whereas our study showed positive bias of difference. However, visual inspection of the Bland-Altman plots from that study might indicate a positive bias during a moderate hypnosis range of 40 to 60 on the mean axis. In their study, Jensen et al. performed their comparisons throughout the full range of hypnosis from alert state to deep hypnosis to recovery. In contrast, we only analyzed the paired-qEEGi data for the anesthesia maintenance period, excluding the induction and recovery periods because the monitors showed different time delays in providing the indexes during these periods.

There are two limitations of this study. First, we only used one kind of electrosurgical device, and we conducted our analyses at $50 \mathrm{~W}$ of fixed output power for ECT. We presumed that different ECT models or different output power could influence abnormal qEEGi behaviors, but in pilot experiments, a few applications of different ECT blend models or unipolar ECT modes at output power of $30 \mathrm{~W}$ and $70 \mathrm{~W}$ showed the same BIS patterns after more than 1 min of ECT. Second, the two sensors can be attached to patients' foreheads in eight combinations of contralateral and ipsilateral and upper and lower locations, and for this study, we randomly used the four contralateral combinations. Niedhart et al. [22] reported the influence of various placements of BIS sensors on BIS monitor bias and agreement, and BIS monitoring failed to provide consistent intra-patient reproducibility when the sensors were placed in different locations. However, this limitation could only concern the agreement between different sensor application methods apart from the influence of ECT on hypnosis monitors.

Conclusively, the electromagnetic interference of ECT during anesthesia could lead to spurious qEEGi monitoring, and this could provide incorrect hypnotic status values and could result in inadvertent titration of anesthetics. Therefore, it is highly recommended that scrutinized clinical judgment be used when interpreting the qEEGi in combination with other available clinical hypnosis information.

\section{ORCID}

Dae-hee Kim, https://orcid.org/0000-0002-1714-257X

Sang-kee Min, https://orcid.org/0000-0001-9796-1542

\section{References}

1. American Society of Anesthesiologists Task Force on Intraoperative Awareness. Practice advisory for intraoperative awareness and brain function monitoring: a report by the American Society of Anesthesiologists task force on intraoperative awareness. Anesthesiology 2006; 104: 847-64.

2. Cullen DJ, Eger EI 2nd, Stevens WC, Smith NT, Cromwell TH, Cullen BF, et al. Clinical signs of anesthesia. Anesthesiology 1972; 36 : 21-36.

3. Imai A, Steffey EP, Ilkiw JE, Farver TB. Comparison of clinical signs and hemodynamic variables used to monitor rabbits during halothaneand isoflurane-induced anesthesia. Am J Vet Res 1999; 60: 1189-95.

4. Rodriguez RA, Hall LE, Duggan S, Splinter WM. The bispectral index does not correlate with clinical signs of inhalational anesthesia during sevoflurane induction and arousal in children. Can J Anaesth 2004; 51: 472-80.

5. Morimoto Y, Hagihira S, Koizumi Y, Ishida K, Matsumoto M, Sakabe T. The relationship between bispectral index and electroencephalographic parameters during isoflurane anesthesia. Anesth Analg 2004; 98: 1336-40.

6. Ekman A, Lindholm ML, Lennmarken C, Sandin R. Reduction in the incidence of awareness using BIS monitoring. Acta Anaesthesiol Scand 2004; 48: 20-6.

7. Punjasawadwong Y, Boonjeungmonkol N, Phongchiewboon A. Bispectral index for improving anaesthetic delivery and postoperative recovery. Cochrane Database Syst Rev 2007; (4): CD003843.

8. Recart A, Gasanova I, White PF, Thomas T, Ogunnaike B, Hamza M, et al. The effect of cerebral monitoring on recovery after general anesthesia: a comparison of the auditory evoked potential and bispectral index devices with standard clinical practice. Anesth Analg 2003; 
97: 1667-74.

9. Jensen EW, Valencia JF, López A, Anglada T, Agustí M, Ramos Y, et al. Monitoring hypnotic effect and nociception with two EEG-derived indices, qCON and qNOX, during general anaesthesia. Acta Anaesthesiol Scand 2014; 58: 933-41.

10. Dahaba AA. Different conditions that could result in the bispectral index indicating an incorrect hypnotic state. Anesth Analg 2005; 101: 765-73.

11. Hemmerling TM, Desrosiers M. Interference of electromagnetic operating systems in otorhinolaryngology surgery with bispectral index monitoring. Anesth Analg 2003; 96: 1698-9.

12. Chan MT, Ho SS, Gin T. Performance of the bispectral index during electrocautery. J Neurosurg Anesthesiol 2012; 24: 9-13.

13. Bland JM, Altman DG. Statistical methods for assessing agreement between two methods of clinical measurement. Lancet 1986; 1: 307-10.

14. Bennett C, Voss LJ, Barnard JP, Sleigh JW. Practical use of the raw electroencephalogram waveform during general anesthesia: the art and science. Anesth Analg 2009; 109: 539-50.

15. Gallagher JD. Pacer-induced artifact in the bispectral index during cardiac surgery. Anesthesiology 1999; 90: 636.

16. Guignard B, Chauvin M. Bispectral index increases and decreases are not always signs of inadequate anesthesia. Anesthesiology 2000; 92: 903.

17. Hemmerling TM, Fortier JD. Falsely increased bispectral index values in a series of patients undergoing cardiac surgery using forced-airwarming therapy of the head. Anesth Analg 2002; 95: 322-3.

18. Kim SH, Lee BC, Kim YH. Falsely increased bispectral index values by convective air warming system during kidney transplantation. Pak J Med Sci 2016; 32: 793-5.

19. Hemmerling TM, Migneault B. Falsely increased bispectral index during endoscopic shoulder surgery attributed to interferences with the endoscopic shaver device. Anesth Analg 2002; 95: 1678-9.

20. Kang MH, In CB, Kim MH, Lim KJ, Park EY, Lee HM, et al. Inappropriate elevation of bispectral index values in robot assisted thyroidectomy with electromyographic endotracheal tube -A case report-. Korean J Anesthesiol 2011; 61: 511-4.

21. Johansen JW. Update on bispectral index monitoring. Best Pract Res Clin Anaesthesiol 2006; 20: 81-99.

22. Niedhart DJ, Kaiser HA, Jacobsohn E, Hantler CB, Evers AS, Avidan MS. Intrapatient reproducibility of the BISxp monitor. Anesthesiology 2006; 104: 242-8. 